\title{
Attentional enhancement in matching-to-sample: Facilitation in matching acquisition by sample-discrimination training
}

\author{
PETER J. URCUIOLI and JUDY CALLENDER \\ Purdue University, West Lafayette, Indiana
}

\begin{abstract}
In three experiments, we examined how matching-to-sample by pigeons is affected by discrimination versus nondifferential training between the matching stimuli. In Experiment 1A, pigeons responding differentially to the sample stimuli off-baseline acquired accurate matching performances more rapidly than did pigeons responding nondifferentially to those same stimuli. In Experiment $1 \mathrm{~B}$, tests involving reversal of the off-baseline requirements demonstrated that the birds were primarily controlled in their matching choices by the sample stimuli. The results of Experiment 2 showed that off-baseline nondifferential training did not retard acquisition relative to comparable training between stimuli unrelated to the matching task. Together, these results suggest that discrimination training can facilitate matching acquisition by enhancing attention to the sample stimuli.
\end{abstract}

Previous studies have shown that pigeons will learn conditional discriminations such as matching-to-sample (MTS) more rapidly if they respond differentially to the conditional (sample) stimuli than if they respond nondifferentially to them (Cohen, Looney, Brady, \& Aucella, 1976; Eckerman, 1970; Lydersen \& Perkins, 1974; Paul, 1983; Urcuioli, 1984; Zentall, Hogan, Howard, \& Moore, 1978). For instance, Cohen et al. (Experiment 2) reported that birds pecking one sample on a differentialreinforcement-of-low-rates-of-responding (DRL) schedule and the other on a fixed-ratio (FR) schedule reached high levels of matching accuracy sooner than did controls whose sample-response requirements were the same for both stimuli. One interpretation of this effect and others like it is that discrimination training enhances subjects' attention to the relevant characteristics of the samples (cf. Carter \& Eckerman, 1975).

My studies of the effects of sample-discrimination training on MTS performance, however, suggest a different interpretation. I found, for example, that pigeons responding differentially to sample stimuli are primarily controlled in their matching choices by their sample-specific behaviors (Urcuioli \& Honig, 1980). This suggests that differential sample behaviors might facilitate acquisition simply by providing birds with an additional cue for choice (see also Cohen, Brady, \& Lowry, 1981; Hogan, Zentall, \& Pace, 1983), an interpretation supported by the results of two subsequent studies. In one study (Urcuioli, 1985), matching acquisition was faster in a group for

This research was supported by NIMH Grant MH-38174 and NSF Grant BNS 86-06926 to the first author. We thank Cynthia Adamiec for her assistance in running the subjects. Reprint requests may be addressed to the first author, Department of Psychological Sciences, Purdue University, West Lafayette, IN 47907. which differential sample responding was correlated with correct choice than in a group for which such responding was uncorrelated with choice. Thus, with sample discriminations equated, the cue properties of the birds' samplespecific behaviors were sufficient to facilitate acquisition. In the other study (Urcuioli, 1984), differential sample responding appeared to reduce rather than enhance conditional stimulus control by the samples. This was indicated by the finding that birds initially trained with differential sample-response requirements (DRL vs. FR) matched at lower levels of accuracy and were slower to recover accurate performances when they were subsequently shifted to a nondifferential, single-peck requirement than controls that were initially trained with either the DRL or the FR requirement. Apparently, the cue arising from DRL versus FR sample responding overshadowed control over choice by the samples themselves (see also Urcuioli, 1985).

Together, then, these earlier results demonstrate that on-baseline discrimination training does not enhance sample-stimulus control over choice when differential sample responding provides a redundant, conditional cue for matching. Still, an enhancement effect might very well occur if birds receive discrimination training separately from MTS acquisition (i.e., off-baseline). In such a procedure, choices would presumably be governed by the discriminated samples themselves and not by any differential responding to them. To date, no one has tested this prediction in tasks such as MTS, although the literature on the acquired distinctiveness of cues (e.g. , Lawrence, 1949, 1950; Mumma \& Warren, 1968) and on the general effects of discrimination training in free-operant situations (e.g., Bresnahan, 1970; Eck, Noel, \& Thomas, 1969; Honig, 1969; Jenkins \& Harrison, 1960; Switalski, Lyons, \& Thomas, 1966; Turner \& Mackintosh, 1972) antici- 
pates just such an effect in conditional as well as in simple discriminations.

\section{A Preliminary Experiment}

In an initial experiment designed to test this prediction, three groups of birds were trained on MTS with lineorientation samples and comparisons. Prior to MTS acquisition, one group received successive discrimination training between the line stimuli. They could obtain food by completing a DRL 3-sec schedule in the presence of one orientation and an FR 10 schedule in the presence of the other. The two remaining groups received nondifferential pretraining in which the same response pattern (either DRL or FR) was reinforced in the presence of both stimuli. During subsequent acquisition of the MTS task, these requirements were replaced for all three groups by a nondifferential, single-peck requirement for producing the comparisons. The expectation was that pigeons that had received prior sample-discrimination training would learn MTS more rapidly than would the nondifferential controls, and that their facilitated performances would reflect more rapid acquisition of conditional stimulus control by the samples. In fact, the discrimination group did acquire MTS at a slightly faster rate than did the two nondifferential groups, but the effect was quite small and the group $\times$ session interaction was only marginally significant.

The effect of discrimination training in this preliminary study may have been small because there were relatively few subjects per group $(n=4)$. But it is also conceivable that the beneficial effects of such training had been counteracted by the shift to the nondifferential, single-peck requirement during MTS acquisition. After all, Honig (1974) showed that nondifferential response training following discrimination training in a free-operant task reduces stimulus control along the previously discriminated dimension (as indicated by flattening of the generalization gradient). Considering his finding, it was hypothesized that continuation of off-baseline sample-discrimination training during MTS acquisition would produce a much larger effect than was seen in the preliminary experiment.

\section{EXPERIMENT 1A}

In this experiment, pigeons received successive discrimination or nondifferential training between the stimuli later serving as samples in MTS. Unlike the preliminary study, however, this training was conducted both prior to and during MTS acquisition, albeit in an off-baseline fashion. Because discrimination versus nondifferential training was now continued during the acquisition phase, additional procedural precautions were implemented to minimize carryover of off-baseline responding to the matching trials themselves.

\section{Method}

Subjects. Twelve experimentally naive White Carneaux pigeons obtained from the Palmetto Pigeon Plant (Sumter, SC) served as subjects. All birds were retired breeders and were housed in in- dividual cages in a colony room with a 14:10 light:dark cycle. During the course of the experiment, they were maintained at $80 \%-85 \%$ of their free-feeding weights by restricted feeding, although grit and water were freely available in the home cage. Prior to the start of the experiment, the birds were randomly assigned to one of the three groups described below.

Apparatus. The experiment was run in a standard BRS/LVE pigeon chamber equipped with a PIP-016 three-key intelligence panel. Mounted behind each of the three keys was a BRS/LVE inline unit (IC-901-IDD) which could project three white vertical and horizontal lines on black backgrounds, and red, blue, green, and white homogeneous fields, onto any response key. General chamber illumination was provided by a partially covered houselight (GE 1829 bulb) mounted $7.6 \mathrm{~cm}$ above the center key. A blower fan mounted on the side of the chamber ran continuously during the experimental sessions to provide forced ventilation and to help mask extraneous noise. This equipment was located in a soundattenuating room along with a Cromemco Z-2D microcomputer for process control.

Procedure. The birds were initially trained to eat reliably out of the food hopper, after which their keypecks to a white centerkey stimulus were shaped by the method of successive approximations. Next, pecking to vertical and horizontal lines was established, and this was followed by two 60-trial sessions of reinforced pecking at these stimuli whenever they appeared on the left, center, or right response keys.

Ten sessions of successive discrimination or nondifferential response training with the vertical and horizontal center-key stimuli followed preliminary shaping. For the birds in Group DF, pecking one line orientation was reinforced on a DRL 3-sec schedule and pecking the other was reinforced on an FR schedule, counterbalanced across subjects. The birds in Group DRL obtained food by pecking both vertical and horizontal lines on the DRL 3-sec schedule; the birds in Group FR had to complete the same FR requirement. The FR requirement for all birds exposed to this contingency was gradually raised from 3 to 10 over the first four sessions, whereas the 3-sec DRL parameter was in effect from the outset for all birds exposed to that contingency. Each line stimulus appeared 30 times during each of the first five pretraining sessions with the restriction that neither stimulus could appear more than three times in succession. The duration of food reinforcement for completing the appropriate schedule requirement was constant within each session but varied from 2.0-3.5 sec for individual birds across sessions to maintain body weights within $80 \%-85 \%$ of their freefeeding weights. Successive trials were separated by a 10-sec intertrial interval (ITD, and the houselight was on continuously throughout each session.

The procedure for the last five pretraining sessions was modified slightly to prepare the birds for the mixture of trial types they would subsequently experience during MTS. During these sessions, 10 of the 60 trials began with a red "ready" stimulus on the center key. The first peck to red after 500 msec turned this stimulus off and, $\mathbf{5 0 0} \mathrm{msec}$ later, produced either the vertical or the horizontal lines on the same key. The first peck to vertical or horizontal after 500 msec then produced food. The remaining 50 trials were identical to those described above for the first five sessions. The purpose of having the red stimulus precede the vertical and horizontal lines on the 10 "single-peck" trials was to establish red as a signal for different upcoming contingencies than those in effect when the lines appeared immediately at the beginning of a trial. The singlepeck trials were distributed randomly throughout each session, with the restrictions that five occur within each successive block of 30 trials and that vertical and horizontal appear equally often after red.

Following the last pretraining session, MTS acquisition with vertical and horizontal samples and comparisons was begun. Each of the 30 MTS sessions consisted of 80 matching trials and 20 simple discrimination or nondifferential trials, depending upon group assignment. For all birds, a MTS trial began with the red "ready" 
stimulus on the center key. The first peck to red after $500 \mathrm{msec}$ turned it off and, 500 msec later, produced either a vertical or a horizontal sample on the center key. A single keypeck at least $500 \mathrm{msec}$ after sample presentation turned off the sample and immediately produced vertical and horizontal comparison stimuli on the adjacent side keys (a zero-delay procedure). Pecking either comparison stimulus turned both off and produced food if the matching comparison was pecked, or an equivalent timeout period with the houselight off if the nonmatching comparison was pecked. The next trial then began following a 10-sec ITI.

On the remaining 20 trials in each MTS session, vertical and horizontal lines (instead of red) appeared on the center key at the start of a trial. For the birds in Group DF, responding differentially (viz., DRL vs. FR) to these lines produced food as during pretraining. Similarly, for the birds in Groups DRL and FR, food was obtained on these trials by completing their respective nondifferential requirements. Four simple discrimination or nondifferential trials appeared randomly in each successive block of 20 trials, two with vertical lines and two with horizontal lines. The remaining 16 MTS trials in each block were divided equally among each of the four possible three-key configurations of samples and comparisons. As during pretraining, reinforcer duration was adjusted individually for each bird across sessions to maintain reduced body weights. The houselight remained on throughout each session with the exception of timeout periods following incorrect matching choices.

Statistical analyses of the data followed the procedure described by Rodger (1975a, 1975b). The tabled $F$ values reported by Rodger (1975a) were used to judge statistical significance. These values control Type I error rate on a per-decision basis by ensuring that the expected proportion of all nulls rejected in error will not exceed $E \alpha$. For all analyses reported in this paper, $E \alpha=05$.

\section{Results and Discussion}

Differential responding to vertical and horizontal in Group DF and nondifferential responding in Groups DRL and FR were quickly established during pretraining. The spaced pattern of responding typical of DRL schedules and the rapid, uninterrupted response pattern typical of FR schedules were also maintained throughout MTS on trials in which food could be obtained by completing the appropriate schedule requirement. On the single-peck trials, the birds typically pecked in rapid fashion throughout the entire sequence of the red "ready" stimulus, the interstimulus interval, and the vertical or horizontal trial stimulus to obtain food during pretraining and the comparisons during MTS. There was no evidence of carryover of DRL-like or FR-like performances to these trials by the start of, or during, MTS acquisition.

Figure 1 shows MTS acquisition for each group over successive two-session blocks of training. Matching accuracy increased in all three groups as training progressed, although the rate of acquisition was more rapid and the terminal level of performance was higher in Group DF than in the two nondifferential groups.

Statistical analysis of these data confirmed the overall between-group difference in performance $[F(2,9)=$ $3.66]$, the improvement over sessions in performance averaged across groups $[F(14,126)=13.08]$, and the interaction between groups and sessions $[F(28,126)=$ 1.03]. Post hoc contrasts across the group means showed that overall performance in Group DF was more accurate than in Group DRL $[F(2,9)=3.59]$, and the averaged

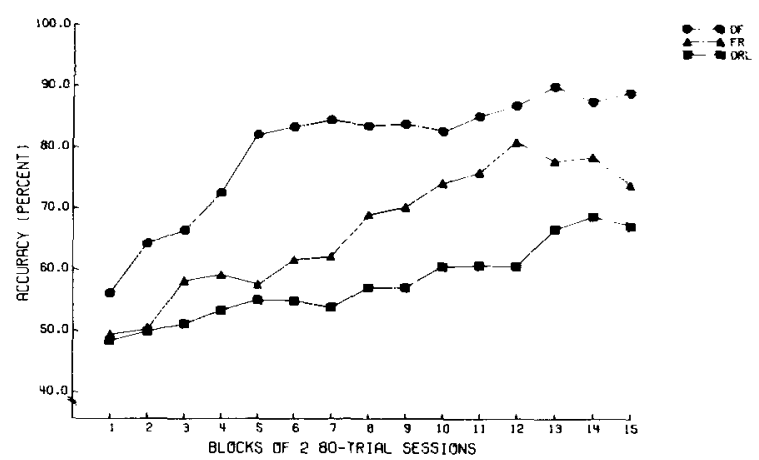

Figure 1. Percentages of correct-choice responses for each group in Experiment 1A averaged over successive blocks of two 80-trial sessions of matching-to-sample acquivition. DF represents off-baseline differential sample-response training prior to and concurrent with acquisition. FR and DRL represent corresponding nondifferential training with fixed-ratio and differential reinforcement response requirements, respectively.

performance in Group FR was in between those of the other two groups $[F(2,9)=.05]$. These statistical decisions imply that matching accuracies averaged across all training sessions were ordered from highest to lowest as follows: Group DF, Group FR, Group DRL (see Rodger, 1975b, Equation 23).

These data demonstrate, then, that off-baseline discrimination training between the samples facilitate the rate at which pigeons learn MTS relative to nondifferentially trained controls. One interpretation of this finding is that discrimination training enhanced attention to the samples and thus speeded the formation of the relevant samplecomparison associations. However, despite the procedural precautions that were taken, another interpretation is that some aspect(s) of DRL and FR responding had in fact carried over from the off-baseline trials to the MTS trials in Group DF, providing those birds with an additional cue for choice (cf. Urcuioli, 1985). Although periodic observations of the birds at the end of the pretraining phase and during MTS acquisition did not reveal any carryover, it is conceivable that differential sample responding was simply too subtle to be easily detected. In the next experiment, we attempted to differentiate between this account and the enhancement interpretation by explicitly testing for control by sample-specific behaviors.

\section{EXPERIMENT 1B}

One method for determining if the choices of the Group DF birds were being cued by some subtle carryover of DRL and FR responding is to reverse the response patterns outside of the matching context, and then to return the birds either to MTS or to the complementary task-oddity-from-sample (OFS). If the choices had been predominantly controlled by differential sample responding, then the reversal should (1) cause accuracy to drop to a level below chance for birds returned to MTS (because the relationship between differential responding and correct choice will also be reversed), and (2) yield 
above-chance levels of accuracy for birds tested on OFS (because the additional switch in correct comparisons will maintain the relationship between sample responding and choice; cf. Urcuioli \& Honig, 1980, Experiment 1). On the other hand, if discrimination training had truly enhanced conditional stimulus control by the samples, then Group DF birds tested on MTS should show above-chance levels of accuracy, whereas those tested on OFS should match at accuracies below chance.

In addition to testing the Group DF birds in this fashion, some of the nondifferential birds from Experiment $1 \mathrm{~A}$ were also tested (viz., by shifting from DRL to FR, or vice versa) to assess the general effects of changing the off-baseline schedules and to verify the presumed control by the sample stimuli in these birds.

\section{Method}

Subjects and Apparatus. Three of the 4 Group DF birds, 2 of the 4 Group FR birds, and 1 Group DRL bird served in this experiment. All were consistently performing at accuracies of $80 \%$ correct or better at the end of Experiment $1 \mathrm{~A}$. The remaining birds from Experiment 1A were not tested either because their terminal levels of matching accuracy were low or because their session-tosession performances were erratic. The apparatus was the same as that described previously.

Procedure. Immediately after completing acquisition in Experiment $1 \mathrm{~A}$, the birds were returned to the pretraining phase for six sessions to reverse the off-baseline response patterns. The first five sessions consisted of 60 discrete trials with the vertical and horizontal center-key lines, but with the DRL and/or FR requirements opposite to what they had previously been. The FR requirement for birds exposed to this contingency was lowered to 3 for the initial session, raised to 5 for the next two sessions, and raised to 10 for all sessions after that. The 3-sec DRL parameter was in effect from the outset of reversal training. The sixth and final session consisted of 50 reversal trials plus 10 additional trials on which single pecks to the vertical and horizontal lines were reinforced. The red "ready" stimulus again preceded the lines on these trials. All other procedural details were identical to those described for pretraining in Experiment $1 \mathrm{~A}$.

Following the last reversal session, all 3 nondifferential birds and 2 of the Group DF birds were tested on MTS. The contingencies and general procedure for the MTS test condition were the same as those described in Experiment $1 \mathrm{~A}$ with the exception that the off-baseline DRL and/or FR requirements were now reversed. The remaining Group DF bird was tested on OFS: for this bird, choice of the nonmatching comparison following each sample was now reinforced. Otherwise, all other procedural details were identical to those for MTS.

\section{Results and Discussion}

Table 1 shows the individual test performances on MTS and OFS following reversal of the DRL and/or FR requirements. Each bird's matching accuracy averaged over the last two sessions of MTS acquisition ("prereversal") is also shown for comparison.

The 2 Group DF birds tested on MTS exhibited positive transfer of performance, matching at accuracy levels well above chance during initial testing. Two of the 3 nondifferential birds tested on MTS showed the same result following the change from DRL to FR, or vice versa. The remaining nondifferential bird (FR 3) fell to chance levels of accuracy but quickly recovered with continued testing
Table 1

Correct Choices Averaged over the Last Two Sessions of Acquisition (Prereversal) and over the First Two Test Sessions of Matching-to-Sample (MTS) or Oddity-from-Sample

(OFS) (Postreversal) Following Reversal of the Off-Baseline Differential or Nondifferential Response Requirements in Experiment 1B

\begin{tabular}{clcc}
\hline Test & Bird & Prereversal & Postreversal \\
\hline MTS & DF 1 & 91.2 & 74.4 \\
& DF 4 & 96.2 & 76.9 \\
& DRL 4 & 78.8 & 78.8 \\
& FR 3 & 78.1 & 56.2 \\
& FR 4 & 99.4 & 73.8 \\
OFS & DF 2 & 98.8 & 28.8 \\
\hline
\end{tabular}

(not shown in the table). All birds except DRL 4 in the MTS test condition performed at lower levels of accuracy during initial testing than at the end of Experiment $1 \mathrm{~A}$. The average drop, however, was similar for the differential and nondifferential subjects $(18.0 \%$ and $15.8 \%$, respectively).

The 1 Group DF bird tested on OFS showed strong negative transfer, choosing the correct comparison on only $21 \%$ and $36 \%$ of all trials, respectively, during Test Sessions 1 and 2 . This result, together with the positive transfer exhibited by the Group DF birds tested on MTS, indicates that matching performances in this group were indeed controlled primarily by the vertical- and horizontalline samples, rather than by any carryover of differential responding to them.

\section{EXPERIMENT 2}

The data from Experiments 1A and 1B are consistent with the view that discrimination training enhances the rate at which sample stimuli acquire control over pigeons' matching choices. However, the acquisition differences could also have arisen because nondifferential response training in the control groups had retarded those birds' learning of the sample-comparison associations. Because the same off-baseline pattern of responding was reinforced in the presence of both samples in Groups DRL and FR, those samples may have been less distinctive than they would have been otherwise. Consequently, they may have been slower to acquire control over choice (cf. Hall \& Channell, 1980; Newlin \& Thomas, 1978; Thomas \& Wheatley, 1974).

To evaluate this latter interpretation, we compared how fast pigeons acquire MTS when off-baseline nondifferential training involves the same stimuli as those used as samples, as opposed to different stimuli. A differentstimulus control was used in preference to a group receiving no off-baseline training because it equates for a variety of factors introduced with such training: the periodic omission of the comparison stimuli on a trial, the absence of the trial-onset cue on a certain proportion of trials/ session, the spacing of matching trials throughout a session, the reinforcement periodically obtained immediately following offset of the center-key stimulus, and so forth. 
If nondifferential response training retards MTS acquisition by producing, say, a stimulus-equivalence effect (Grice, 1966; Thomas, Miller, \& Hansen, 1972), then birds should learn to match more slowly if such training involves stimuli that also appear as samples on the matching trials (intradimensional training) than if it involves different stimuli (extradimensional training) (Hall \& Channell, 1980).

\section{Method}

Subjects. Sixteen experimentally naive White Carneaux pigeons (retired breeders), obtained from the same supplier and housed and maintained in the same fashion as the pigeons in the preceding experiments, served as subjects. They were randomly divided into the four groups described below prior to the start of the experiment. The experiment was run in two replications, with subjects from each group represented in each replication.

Apparatus. The apparatus was the same as that described previously.

Procedure. Preliminary training and pretraining for the subjects assigned to the intradimensional nondifferential (FR-Intra and DRLIntra) groups were virtually identical to those described in Experiment 1A. Vertical and horizontal lines (which later appeared as samples and comparisons in MTS) were present during all sessions of preliminary training and pretraining. The only difference in the treatment of the intradimensional subjects in this experiment compared with those in Experiment 1A was the use of a white stimulus (instead of red) as the trial-onset cue for the single-stimulus trials programmed during the last five sessions of pretraining.

The subjects in the extradimensional nondifferential (FR-Extra and DRL-Extra) groups received their preliminary training and pretraining with blue and green center-key hues instead of with the lines. The 10 single-stimulus trials programmed during each of the last five pretraining sessions, however, were identical to those for the intradimensional birds: single pecks to the vertical and horizontal lines were reinforced following the white trial-onset cue.

Following pretraining, all subjects were run for 30 sessions on MTS with vertical and horizontal lines as samples and comparisons. Each MTS session consisted of 80 matching trials and 20 offbaseline nondifferential trials. The contingencies for the off-baseline trials were identical to those in effect for each group during pretraining. In other words, the birds in Groups FR-Intra and FR-Extra could obtain food by responding on the same FR schedule to vertical and horizontal lines or to blue and green, respectively, whereas

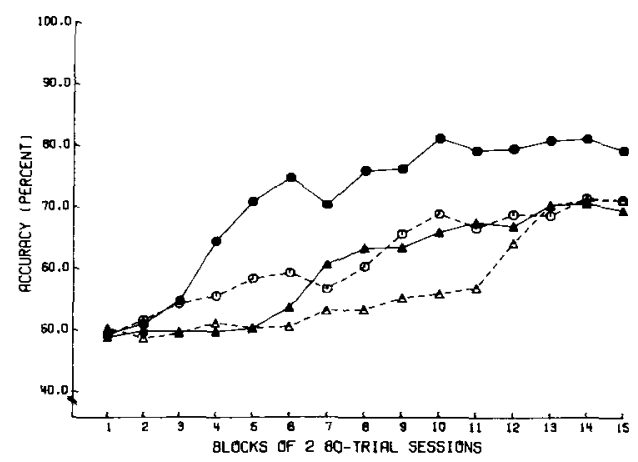

Figure 2. Percentages of correct-choice responses for each group in Experiment 2 averaged over successive blocks of two 80-trial sessions of matching-to-sample acquisition. INTRA represents offbaseline nondifferential training with the sample stimuli. EXTRA represents off-baseline training with stimuli orthogonal to those in the matching task.

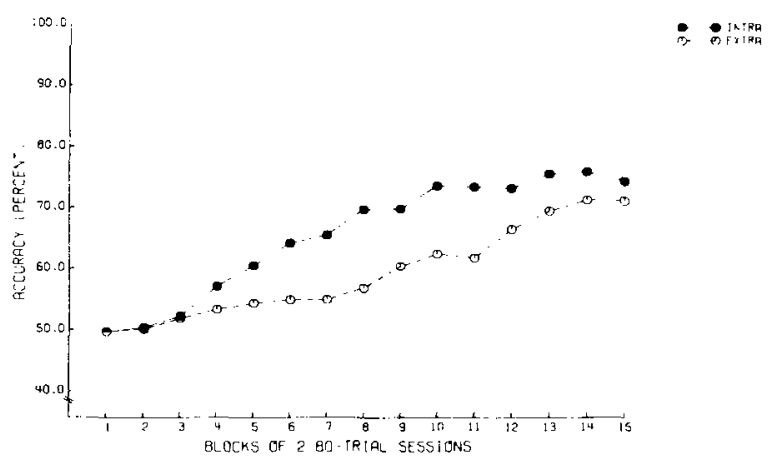

Figure 3. Percentages of correct-choice responses over successive two-session blocks of acquisition in Experiment 2 for the two intradimensional (INTRA) and the two extradimensional (EXTRA) groups combined.

the birds in the two DRL groups (DRL-Intra and DRL-Extra) had the same DRL schedule in effect for both of their stimuli.

\section{Results and Discussion}

Figure 2 shows MTS acquisition for each group over successive blocks of two sessions. The filled symbols plot the functions for the intradimensional groups; the open symbols plot the corresponding functions for the extradimensional groups. Contrary to expectations that intradimensional nondifferential training might retard MTS acquisition, the birds in the two intradimensional groups (FR-Intra and DRL-Intra) showed somewhat more rapid acquisition over the 30 training sessions than did the birds in the two extradimensional groups (FR-Extra and DRLExtra). Analysis of variance, however, revealed that only the effect of training sessions was significant $[F(14,196)$ $=14.45]$. Neither the group effect nor the group $\times$ session interaction was significant.

Still, these results demonstrate that intradimensional nondifferential training definitely did not slow the rate of MTS acquisition relative to that of the extradimensional controls. Figure 3 makes this clearer by plotting performances averaged over both intradimensional and both extradimensional groups. The average level of performance for the intradimensional subjects was higher throughout most of training than for the extradimensional subjects although, again, the overall group effect was not significant $[F(1,14)=1.18]$. Still, accuracy did improve significantly with training, and at different rates in these two conditions $\left[F(14,196)=14.45\right.$ and $0.98^{1}$, respectively]

These findings thus support the argument that the previously observed differences in MTS acquisition between groups receiving discrimination versus nondifferential training were primarily due to an enhancing effect of discrimination training, rather than to a retardation effect produced by nondifferential training.

\section{GENERAL DISCUSSION}

The major results and conclusions of the experiments reported in this paper can be summarized as follows. Pigeons given off-baseline discrimination training prior 
to and concurrently with MTS acquired accurate matching performances more rapidly than did nondifferentially trained controls (Experiment 1A). This difference in acquisition reflects a true facilitation effect, because nondifferential training between the sample stimuli did not retard the rate at which the pigeons learned to match (Experiment 2). Finally, the beneficial effects of discrimination training appear to be attributable to enhanced conditional stimulus control by the samples, as indicated by the finding of positive transfer to MTS and negative transfer to OFS following reversal of the off-baseline schedules (Experiment 1B).

Prior studies of the effects of sample-discrimination training (Cohen et al., 1976; Eckerman, 1970) have also found that such training facilitates conditional discrimination learning. However, in those studies, subjects responded differentially to the samples on the matching baseline to produce the comparisons. Consequently, the additional response-produced cue provided by those differential responses (Urcuioli \& Honig, 1980) can account for the rapid acquisition (Urcuioli, 1985). This confound was avoided in the present study by conducting discrimination training off-baseline. Here, differential "sample" responding was reinforced only when the matching contingencies were not in effect. Although such training did not prohibit the birds from responding differentially to the samples on the matching trials themselves, the reversal tests conducted following acquisition indicated that if such responding had been present, it was not the major source of control over choice. Instead, that function was exercised by the samples themselves.

Of course, the finding that the sample stimuli exerted predominant control over the discrimination birds' matching performances does not rule out the possibility that some subtle form of differential sample responding also contributed to performance. Joint control cannot be detected by opposing-cues transfer tests like those used in Experiment 1B. As a result, all that can be said with certainty at this point is that the samples provided the stronger cue for choice. Still, I have found in other, unpublished experiments that discrimination birds like those trained in Experiment $1 \mathrm{~A}$ perform at high levels of accuracy and are controlled in their choices by the sample stimuli, even when nondifferential birds show no signs of MTS acquisition. Thus, relative to controls, discrimination training must have enhanced control by the samples, in addition to whatever other effects it may or may not have had.

An issue left unanswered by these experiments is whether discrimination training has its primary effect upon attention to the samples or whether attention to the comparisons might also be influenced by such training. Given that Carter and Eckerman (1975) have demonstrated that rates of MTS acquisition are influenced to a larger extent by sample discriminability than by comparison discriminability, and that the location of the discriminated stimuli in Experiment $1 \mathrm{~A}$ was the same as that at which the samples appeared, one would suspect that the major effect of discrimination training is on sample processing. The results of other, unpublished experiments conducted in our laboratory are compatible with this inference: discrimination training facilitated acquisition of symbolic MTS if the discriminated stimuli appeared only as samples but not if they appeared only as comparisons.

The consequence of attentional enhancement is consistent with Cumming, Berryman, and Cohen's (1965) coding analysis of conditional discrimination learning. According to Cumming et al., subjects learn a unique coding response to each sample during MTS. Choices between the comparisons are then mediated by the coding responses rather than by the physical characteristics of the samples alone (see also Berryman, Cumming, Cohen, \& Johnson, 1965; Eckerman, Lanson, \& Cumming, 1968; Maki, Gillund, Hauge, \& Siders, 1977). According to this argument, the rate at which birds acquire MTS will be determined by how quickly they learn the coding responses to each sample stimulus (Carter \& Werner, 1978), which in turn should be governed by how rapidly they come to attend to the relevant features of the samples. Discrimination training should thus speed the entire process by influencing attentional mechanisms (e.g., Mackintosh, 1977; Thomas, 1970; Wagner, 1969). But no matter what the mechanism underlying enhancement, the "samplesas-coded" (cf. Lawrence, 1963) do not appear to correspond in any straightforward fashion to the differential responses reinforced off-baseline.

\section{REFERENCES}

Berryman, R., Cumming, W. W., Cohen, L. R., \& Johnson, D. F. (1965). Acquisition and transfer of simultaneous oddity. Psychological Reports, 17, 767-775.

BrESNAHAN, E. L. (1970). Effects of extradimensional pseudodiscrimination and discrimination training upon stimulus control. Journal of Experimental Psychology, 85, 155-156.

Carter, D. E., \& ECKerman, D. A. (1975). Symbolic matching by pigeons: Rate of leaming complex discriminations predicted from simple discriminations. Science, 187, 662-664.

Carter, D. E., \& Werner, T. J. (1978). Complex learning and information processing by pigeons: A critical analysis. Journal of the Experimental Analysis of Behavior, 29, 565-601.

Cohen, L. R., Brady, J., \& Lowry, M. (1981). The role of differential responding in matching-to-sample and delayed matching performance. In M. L. Commons \& J. A. Nevin (Eds.), Quantitative analyses of behavior: Discriminative properties of reinforcement schedules (Vol. 1, pp. 345-364). Cambridge, MA: Ballinger.

Cohen, L. R., Looney, T. A., Brady, J. H., Aucella, A. F. (1976). Differential sample response schedules in the acquisition of conditional discriminations by pigeons. Joumal of the Experimental Analysis of Behavior, 26, 301-314.

Cumming, W. W., Berryman, R., \& Cohen, L. R. (1965). Acquisition and transfer of zero-delay matching. Psychological Reports, 17, 435-445.

Eck, K. O., Noel, R. C., Thomas, D. R. (1969). Discrimination learning as a function of prior discrimination and nondifferential training. Journal of Experimental Psychology, 82, 156-162.

ECKERMAN, D. A. (1970). Generalization and response mediation of a conditional discrimination. Journal of the Experimental Analysis of Behavior, 13, 301-316.

Eckerman, D. A., Lanson, R. N., Cumming, W. W. (1968). Acquisition and maintenance of matching without a required observing response. Journal of the Experimental Analysis of Behavior, 11, 435-441 
Grice, G. R. (1966). Investigations of response-mediated generalization. In D. 1. Mostofsky (Ed.), Stimulus generalization (pp. 373-382). Stanford, CA: Stanford University Press.

Hall, G., \& Channell, S. (1980). A search for perceptual differentiation by nondifferential reinforcement. Quarterly Journal of Experimental Psychology, 32, 185-195.

Hogan, D. E., Zentall, T. R., \& Pace, G. (1983). Control of pigeons' matching-to-sample performance by differential sample response requirements. American Journal of Psychology, 96, 37-49.

HoNiG, W. K. (1969). Attentional factors governing the slope of the generalization gradient. In R. M. Gilbert \& N. S. Sutherland (Eds.), Animal discrimination learning (pp. 35-62). London: Academic Press.

HoNIG, W. K. (1974). Effects of extradimensional discrimination training upon previously acquired stimulus control. Learning \& Motivation, 5, $1-15$.

JENKINS, H. M. , HARRISON, R. H. (1960). Effect of discrimination training on auditory generalization. Journal of Experimental Psychology, 59, 246-253.

LAWRENCE, D. H. (1949). Acquired distinctiveness of cues: I. Transfer between discriminations on the basis of familiarity with the stimulus. Journal of Experimental Psychology, 39, 770-784.

LAWRENCE, D. H. (1950). Acquired distinctiveness of cues: II. Selective association in a constant stimulus situation. Joumal of Experimental Psychology, 40, 175-188.

LAWRENCE, D. H. (1963). The nature of a stimulus: Some relationships between learning and perception. In S. Koch (Ed.), Psychology: A study of a science (Vol. 5, pp. 179-212). New York: McGrawHill.

Lydersen, T., \& Perkins, D. (1974). Effects of response-produced stimuli upon conditional discrimination performance. Journal of the Experimental Analysis of Behavior, 21, 307-314.

MackIntosh, N. J. (1977). Stimulus control: Attentional factors. In W. K. Honig \& J. E. R. Staddon (Eds.), Handbook of operant behavior (pp. 481-513). Englewood Cliffs, NJ: Prentice-Hall.

Maki, W. S., Gillund, G., Hauge, G., Siders, W. A. (1977). Matching to sample after extinction of observing responses. Journal of Experimental Psychology: Animal Behavior Processes, 3, 285-296.

Mumma, R., \& WarRen, J. M. (1968). Two-cue discriminatory learning by cats. Journal of Comparative \& Physiological Psychology, 66, 116-122.

Newlin, R. J., \& Thomas, D. R. (1978). Nondifferential training of pigeons retards acquisition of subsequent discriminations involving other stimuli. Animal Learning \& Behavior, 6, 385-390.

PAUL, C. (1983). Sample-specific ratio effects in matching to sample. Journal of the Experimental Analysis of Behavior, 39, 77-85.

RODGER, R. S. (1975a). The number of non-zero, post-hoc contrasts from ANOVA and error rate. I. British Journal of Mathematical \& Statistical Psychology, 28, 71-78.
RODGER, R. S. (1975b). Setting rejection rate for contrasts selected pist hoc when some nulls are false. British Journal of Mathematical \& Statistical Psychology, 28, 214-232.

Switalski, R. W., Lyons, J., Thomas, D. R. (1966). Effects of interdimensional training on stimulus generalization. Journal of Experimental Psychology, 72, 661-666.

TномAS, D. R. (1970). Stimulus selection, attention, and related matters. In J. H. Reynierse (Ed.), Current issues in animal learning (pp. 31 1-356). Lincoln: University of Nebraska Press.

Thomas, D. R., Miller, J. T., \& Hansen, G. (1972). Role of stimulus comparison in equivalence training. Journal of Experimental Psychology, 96, 297-300.

Thomas, D. R., \& Wheatley, K. L. (1974). Effects of interdimensional training on stimulus generalization: An extension. Joumal of Experimental Psychology, 103, 1080-1085.

Turner, C., Mackintosh, N. J. (1972). Stimulus selection and irrelevant stimuli in discrimination learning by pigeons. Joumal of Comparative \& Physiological Psychology, 78, 1-9.

URCUIOLI, P. J. (1984). Overshadowing in matching-to-sample: Reduction in sample-stimulus control by differential sample behavors. Animal Learning \& Behavior, 12, 256-264.

URCUiol, P. J. (1985). On the role of differential sample behaviors in matching-to-sample. Journal of Experimental Psychology: Animal Behavior Processes, 11, 502-519.

Urcuioli, P. J., \& Honig, W. K. (1980). Control of choice in conditional discriminations by sample-specific behaviors. Journal of Experimental Psychology: Animal Behavior Processes, 6, 251-277.

WAGNER, A. R. (1969). Incidental stimuli and discrimination learning. In R. M. Gilbert \& N. S. Sutherland (Eds.), Animal discrimination learning (pp. 83-111). London: Academic Press.

Zentall, T. R., Hogan, D. E., Howard, M. M., Moore, B. S. (1978). Delayed matching in the pigeon: Effect on performance of sample-specific observing responses and differential delay behavior. Leaming \& Motivation, 9, 202-218.

\section{NOTE}

1. Because the expected proportion of Type I errors is held constant on a per-decision basis (at .05 or .01) in Rodger's (1975a) tables, the critical $F$ values obtained by integration of the central $F$ distribution are generally less than 1 when $E \alpha=.05$ and when $\nu_{1}>12$ (see Rodger, 1975a, Table la, and Equations 9 and 10). Consequently, the declared significance of the interaction in this experiment is neither a misprint nor a misinterpretation of the statistical findings by Rodger's criterion.

(Manuscript received September 16, 1988: revision accepted for publication January 19, 1989.) 